\title{
Quality evaluation of different varieties of dry red wine based on nuclear magnetic resonance metabolomics
}

\author{
Boran $\mathrm{Hu}^{1 *}$, Jin Gao ${ }^{1}$, Shaochen Xu1,2, Jiangyu Zhu ${ }^{1}$, Xuemei Fan ${ }^{3}$ and Xiaoyan Zhou ${ }^{1 *}$
}

\begin{abstract}
The metabolites that provide the aroma and flavor to wine are the products of several influences, such as grape cultivar, geographic location and associated environmental features, viticultural practices, and vinification techniques, which are central to production protocols, quality evaluation and development of wine regions. Accordingly, we initiated the requisite studies to investigate the differences in the dry red wine metabolites of different grape varieties. The proton-nuclear magnetic resonance technique ( $\left.{ }^{1} \mathrm{H}-\mathrm{NMR}\right)$ combined with multivariate statistical analysis was used to investigate the changes of metabolite levels in Cabernet Sauvignon, Merlot and Cabernet Gernischt dry red wines vinified in Changli, Hebei province, China, in 2017. The results showed that the types of metabolites in different varieties of dry red wines were similar, but the content was significantly different. The main contributors to the differences in Cabernet Sauvignon, Merlot and Cabernet Gernischt dry red wines were ethyl acetate, lactic acid, alanine, succinic acid, proline, malic acid, and gallic acid, indicating ${ }^{1} \mathrm{H}-\mathrm{NMR}$ method combined with multivariate statistical analysis can distinguish these three types of dry red wines from each other. It provides a benchmark for further comparative study on wine quality and the verification of wine authenticity.
\end{abstract}

Keywords: Proton nuclear magnetic resonance technology, Metabolites, Multivariate statistical analysis, Wine varieties

\section{Introduction}

Dry red wine is a kind of natural alcoholic beverage with health care function. Long-term moderate drinking can delay aging, prevent and inhibit cardiovascular disease and cancer. As dry red wine contains colored substances in the peel or pulp, it is mainly in red color, such as deep ruby red, ruby red, magenta, crimson, brown red and so on. Changli is one of the important wine grape producing areas in China, with superior geographical location, unique geomorphological features, good soil and meteorological ecological conditions. Changli is located at $39^{\circ}$ $24^{\prime}-40^{\circ} 37^{\prime}$ north latitude, which is the same latitude as

\footnotetext{
*Correspondence: huboran@yzu.edu.cn; Yzuxyz@163.com

${ }^{1}$ The School of Tourism and Colinary Arts (The School of Food Science and Engineering), Yangzhou University, 196 Huayang West Road, Yangzhou 225127, Jiangsu, China

Full list of author information is available at the end of the article
}

Bordeaux, France. The unique geographical characteristics of bordering the Bohai Sea in the East and Yanshan Mountain in the North have created Changli production area. The annual sunshine duration is $2600-2800 \mathrm{~h}$, the mean temperature difference between day and night is 12 degrees, and the annual rainfall is $400-600 \mathrm{~mm}$. Therefore, it is necessary to study the metabolites of dry red wine in Changli region. Meanwhile, with the continuous development of the wine market, red wine counterfeiting and adulteration incidents have also occurred. Considering consumers' pursuit of wine quality and safety, it is significant to find a convenient and fast way to identify different kinds of dry red wines, which provides a certain reference for consumers to choose dry red wine.

Nuclear magnetic resonance (NMR) technology has become an important detection method to obtain structural information of metabolites because of its fast analysis speed, simple sample preparation, good repeatability 
and good stability [1]. The combination of ${ }^{1} \mathrm{H}$ NMR and pattern recognition technology has been widely used not only in food industry, but also in wine and some liquid beverages such as oil, juice, green tea, beer, etc. [2-5]. Ali et al. [6] used NMR technology combined with pattern recognition analysis to study the inhibitory effect of wine metabolites on tumor necrosis factor. The results showed that the inhibitory effect of wine on tumor necrosis factor was affected by the influence of the vintage and type of wine on the metabolites. Mazzei et al. [7] used NMR techniques to analyze the wines from vineyards with different climatic and soil characteristics in Campania, Italy. The results showed that the differences between wines are associated with climate, the content of carbonate and organic matter in soil and other factors. As a commonly used data analysis method, multivariate statistical method is often combined with NMR technology, and is widely used in the determination and classification of food sources [8-11]. Lee et al. [12] used NMR techniques and multivariate statistical analysis method to distinguish wines from different vintages. The results showed that the main compounds causing differences in wines in different years were 2,3-butanediol, lactic acid, alanine, proline, $\gamma$-aminobutyric acid, choline, and polyphenols. Zhu et al. [13] used ${ }^{1} \mathrm{H}$-NMR combined with pattern recognition technology and multivariate statistical analysis method to make a distinction between Cabernet Sauvignon and Shiraz red wine brewed in Shanxi in 2016, and found key contributors to differences were proline, tartaric acid, glycerol, lactic acid, choline, succinic acid and gallic acid. All these results showed that ${ }^{1} \mathrm{H}-\mathrm{NMR}$ combined with multivariate analysis was a good tool for identifying the different wines.

This study investigated the differences of metabolites in different varieties of dry red wine based on ${ }^{1} \mathrm{H}-\mathrm{NMR}$ metabolomics and multivariate statistical analysis methods, provided an effective and feasible method for the identification of dry red wine varieties, and offered reasonable advice for consumers to choose suitable dry red wines.

\section{Materials and methods Wine sample}

The wines used in this experiment were provided by Hebei Changli Grape Wine Co., Ltd., and all the single varieties of wine in 2017 were made by the standard process, using the following techniques: De-stemmed and crushed the grapes, and then added the yeast to ferment at $25{ }^{\circ} \mathrm{C}$ for $8-10$ days, followed by pressing the pomace gently. The wine was separated and tank-switched, and then sampled for pretreatment. The physical and chemical indicators of all wine samples were in line with the requirements of the Chinese
National Standard (GB15037-2006), and the samples were stored at $-4{ }^{\circ} \mathrm{C}$ for further use.

\section{NMR spectroscopic analysis NMR sample pretreatment}

Ten milliliters of wine were taken, and then centrifuged at the speed of $3000 \mathrm{rpm}$ at $-4{ }^{\circ} \mathrm{C}$ for $20 \mathrm{~min}$. Three milliliters of supernatant fluid were pre-frozen at $-80{ }^{\circ} \mathrm{C}$ for $12 \mathrm{~h}$, and then lyophilized for $48 \mathrm{~h}$. The lyophilized product was dissolved in $400 \mu \mathrm{L}$ of oxalate buffer $(\mathrm{pH}=4$; Shanghai Suyi Chemical Reagent Co., Ltd., China), $140 \mu \mathrm{L} \mathrm{D} \mathrm{D}_{2} \mathrm{O}$ (deuterated degree $>99.9 \%$; Qingdao Tenglong Microwave Technology Co., Ltd., China) and $60 \mu \mathrm{L}$ of $0.5 \%$ DSS (4,4-dimethyl-4-silapentanesulfonate), the mixture were centrifuged at the speed of $13,000 \mathrm{rpm}$ for $20 \mathrm{~min}$. At last, $500 \mu \mathrm{L}$ of the supernatant was loaded into a $5 \mathrm{~mm}$ nuclear tube for NMR analysis. Each sample was tested 4 times.

\section{NMR spectrum acquisition}

${ }^{1} \mathrm{H}$-NMR spectra of wine samples were taken on an AVANCE 600 Nuclear magnetic resonance Spectrometer (Bruker Co., Ltd., Germany). The experiments were carried out at a constant temperature of $298 \mathrm{~K}$. The ${ }^{1} \mathrm{H}-\mathrm{NMR}$ operating frequency was $600.23 \mathrm{MHz}$ and the spectral width was $7183.9 \mathrm{~Hz}$. The number of sampling points was $32 \mathrm{k}$. The relaxation delay was set to $2 \mathrm{~s}$ and the sampling time was set to $2.3 \mathrm{~s}$. The linewidth enhancement factor was $0.3 \mathrm{~Hz}$. The NOESYGPPR1D sequence was used to suppress the water peak signal, and the number of scans was set at 256 times.

\section{NMR spectral data processing}

The chemical shift interval between 0 and $10.0 \mathrm{ppm}$ in NMR spectrum was integrated at the section of $0.005 \mathrm{ppm}$ by using Software AMIX. The DSS peaks of $-0.5-0.5 \mathrm{ppm}, 1.74-1.84 \mathrm{ppm}$, and $2.90-2.95 \mathrm{ppm}$, the residual ethanol peaks of 1.18-1.22 ppm and 3.57$3.72 \mathrm{ppm}$, and the residual water peak of 4.8-4.96 ppm were removed. The integral data obtained by nuclear magnetics was normalized and then imported into Software SIMCA-P 12.0 for multivariate statistical analysis. In order to establish a more reasonable regression model, partial least square discriminant analysis (PLSDA) was used to strengthen the separation between the observation groups. In addition, PLS-DA also helps to understand which components carry category separation information, and the fitting degree of PLS-DA is verified by external model verification experiments. 


\section{Results}

\section{Identification of metabolites in wine}

One-dimensional NMR spectrum can provide structural information of metabolites. The NMR spectra of Cabernet Sauvignon, Merlot and Cabernet Gernischt wines were shown in Fig. 1. As seen from the figure, most of the metabolites in the three wines were concentrated in the range of $\delta \mathrm{H} 9.00-\delta \mathrm{H} 0.00$. According to the related literature [14-17], the main characteristic peaks in the NMR spectrum of the corresponding wines were assigned, and the results were shown in Table 1.

It can be seen from the identified metabolites that these substances mainly included amino acids, organic acids, sugar, and phenolic, etc. These metabolites represented the overall metabolome of wine, indicating that ${ }^{1} \mathrm{H}$-NMR can analyze these metabolites synchronously with high throughput [18]. At the same time, it could be found that most of the small molecule metabolites in wine samples were the same, which meant that the composition of small molecule metabolites in wine was relatively stable. However, the contents of metabolites in different types of wine samples were different, and each type of wine had its own characteristic spectrum, indicating that metabolic spectrogram in different types of wine samples described their physiological and biochemical states. Therefore, it is necessary to process these spectral data to find out the markers. The characteristic peaks in the NMR spectrum correspond to different metabolites in the wine samples, and the peak intensity (such as peak area) represented the relative content of the corresponding metabolites [19]. Consequently, the NMR spectrum can effectively show the composition and content of metabolites in the wine samples, and can be studied as a metabolic fingerprint.

\section{Differences in metabolites between different varieties of dry red wine}

To find out the main metabolites that cause the differences in different types of dry red wine, NMR data of the dry red wine samples were imported into SIMCA P-12.0 software for Partial least squares discrimination analysis (PLS-DA). The PLS-DA model was established and the scores plot, cross-validation plot and loading plot of Merlot and Cabernet Sauvignon dry red wine was shown in Fig. 2. As seen from the scores plot, the two types of dry red wines could be clearly separated on the PC1 axis, and the cumulative contribution rate $R^{2} X=0.935$, $\mathrm{R}^{2} \mathrm{Y}=0.999, \mathrm{Q}^{2}=0.996$, indicating that the model was

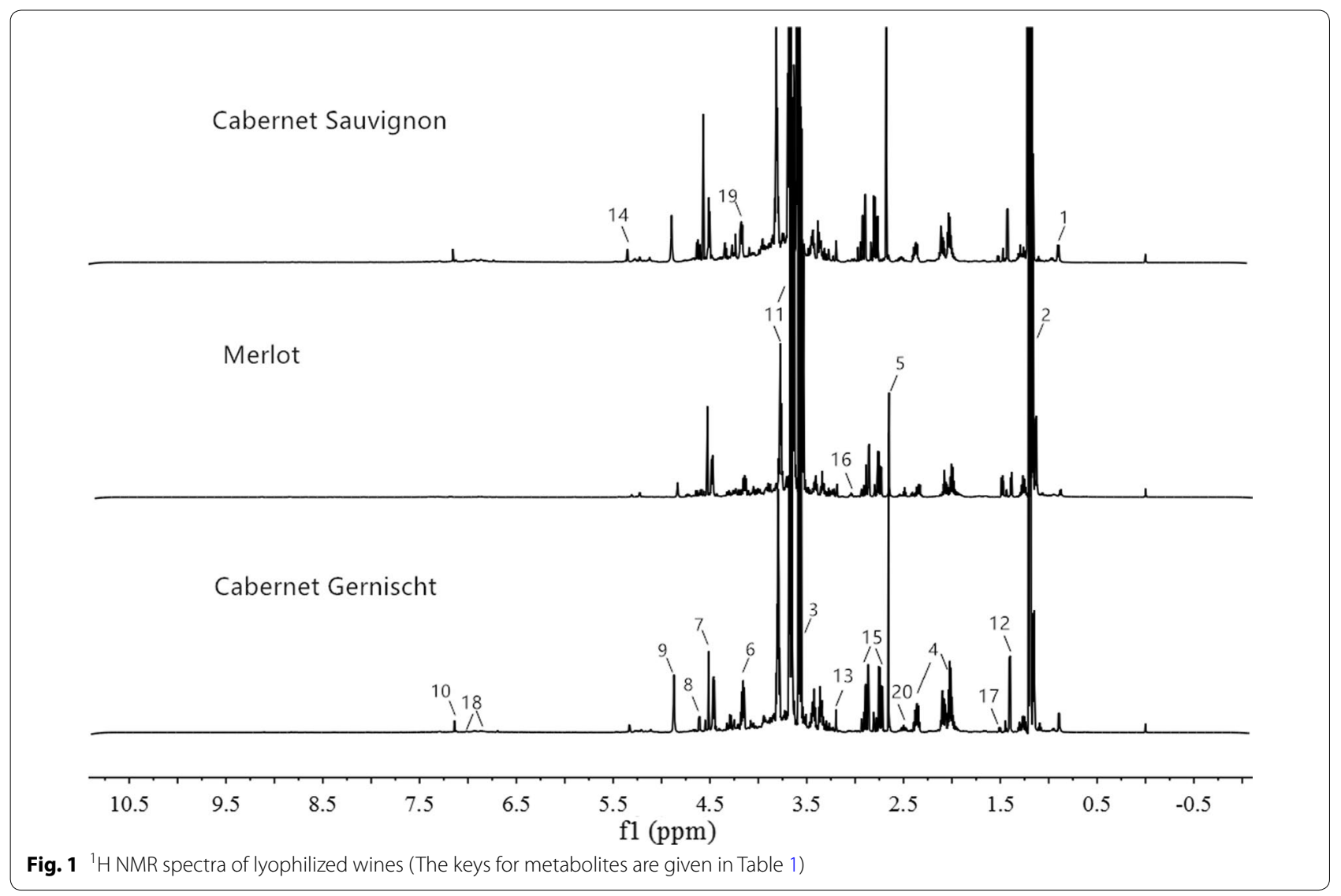


Table $1{ }^{1} \mathrm{H}$ NMR assignment of metabolites in wines

\begin{tabular}{|c|c|c|c|}
\hline Keys & Compound & ${ }^{1} \mathrm{H}-\mathrm{NMR}$ chemical shift & Group \\
\hline 1 & Valine & $0.88(d), 1.02(d)$ & $\mathrm{C}_{4} \mathrm{H}_{3}, \mathrm{C}_{5} \mathrm{H}_{3}$ \\
\hline 2 & 2,3-Butanediol & $1.15(d)$ & $\mathrm{C}_{1} \mathrm{H}_{3}+\mathrm{C}_{4} \mathrm{H}_{3}$ \\
\hline 3 & Ethanol & $1.19(\mathrm{t}), 3.56(\mathrm{q})$ & $\mathrm{C}_{2} \mathrm{H}_{3}, \mathrm{C}_{1} \mathrm{H}_{2}$ \\
\hline 4 & Proline & $\begin{array}{l}2.00(\mathrm{~m}), 2.07(\mathrm{~m}), 2.35(\mathrm{~m}), 3.35(\mathrm{~m}), 3.42(\mathrm{~m}) \\
\quad 4.16(\mathrm{~m})\end{array}$ & $\gamma-\mathrm{CH}_{2}, \beta-\mathrm{CH}, \beta^{\prime}-\mathrm{CH}, \delta-\mathrm{CH}, \delta-\mathrm{CH}, \mathrm{a}-\mathrm{CH}$ \\
\hline 5 & Succinic acid & $2.65(\mathrm{~s})$ & $\mathrm{C} 2 \mathrm{H}_{2}+\mathrm{C}_{3} \mathrm{H}_{2}$ \\
\hline 6 & Ethyl acetate & $1.26(\mathrm{t}), 4.16(\mathrm{q})$ & $\mathrm{C}_{4} \mathrm{H}_{3}, \mathrm{C}_{3} \mathrm{H}_{2}$ \\
\hline 7 & Tartaric acid & $4.51(\mathrm{~s})$ & $\mathrm{C} 2 \mathrm{H}+\mathrm{C} 3 \mathrm{H}$ \\
\hline 8 & $\beta$-Glucose & $4.61(d)$ & $\beta C 1 H$ \\
\hline 9 & a-Glucose & $5.33(d)$ & $\mathrm{aC} 1 \mathrm{H}$ \\
\hline 10 & Gallic acid & 7.14(s) & $\mathrm{C} 2 \mathrm{H}+\mathrm{C} 6 \mathrm{H}$ \\
\hline 11 & Glycerol & $3.58(\mathrm{q}), 3.67(\mathrm{~m}), 3.81(\mathrm{~m})$ & $\mathrm{C} 2 \mathrm{H}_{2}, \mathrm{C}_{3} \mathrm{H}_{2}, \mathrm{C} 1 \mathrm{H}$ \\
\hline 12 & Lactic acid & $1.39(\mathrm{~d}), 4.16(\mathrm{~m})$ & $\mathrm{C}_{3} \mathrm{H}_{3}, \mathrm{C} 2 \mathrm{H}$ \\
\hline 13 & Choline & $3.20(s)$ & $\mathrm{N}-\mathrm{CH}_{3}$ \\
\hline 14 & a-D-Glucuronic acid & $5.35(d)$ & $\mathrm{C} 1 \mathrm{H}$ \\
\hline 15 & Malic acid & $2.74(d d), 2.87(d d), 4.46(q)$ & $\beta \mathrm{CH}_{2}, \beta^{\prime} \mathrm{CH}_{2}, \mathrm{CH}$ \\
\hline 16 & Citric acid & $2.82(d), 2.94(d)$ & $\mathrm{C} 2 \mathrm{H}_{\mathrm{a}}+\mathrm{C}_{4} \mathrm{H}_{\mathrm{a}^{\prime}} \mathrm{C} 2 \mathrm{H}_{\mathrm{b}}+\mathrm{C}_{4} \mathrm{H}_{\mathrm{b}}$ \\
\hline 17 & Alanine & $1.51(d)$ & $\mathrm{\beta CH}_{3}$ \\
\hline 18 & Tyrosine & $6.86(d), 7.19(d)$ & $\mathrm{C} 2 \mathrm{H}, \mathrm{C} 3 \mathrm{H}$ \\
\hline 19 & D-Sucrose & $\begin{array}{l}5.46(\mathrm{~d}), 3.55(\mathrm{dd}), 3.72(\mathrm{dd}), 3.90(\mathrm{dd}), 4.215(\mathrm{~d}) \\
\quad 4.05(\mathrm{dd}), 3.88(\mathrm{dd})\end{array}$ & $\mathrm{C} 1 \mathrm{H}, \mathrm{C} 2 \mathrm{H}, \mathrm{C} 3 \mathrm{H}, \mathrm{C} 4 \mathrm{H}, \mathrm{C} 1^{\prime} \mathrm{H}, \mathrm{C} 2^{\prime} \mathrm{H}, \mathrm{C} 3^{\prime} \mathrm{H}$ \\
\hline 20 & $\gamma$-Aminobutyric acid & $2.50(\mathrm{t}), 1.96(\mathrm{~m}), 3.05(\mathrm{t})$ & $\mathrm{a}-\mathrm{CH}_{2}, \beta-\mathrm{CH}_{2}, \gamma-\mathrm{CH}_{2}$ \\
\hline
\end{tabular}

Letters in parentheses indicate the peak multiplicities: $\mathrm{s}$ (singlet), $\mathrm{d}$ (doublet), $\mathrm{t}$ (triplet), q (quartet), $\mathrm{m}$ (multiplet) and dd (doublet of doubles)

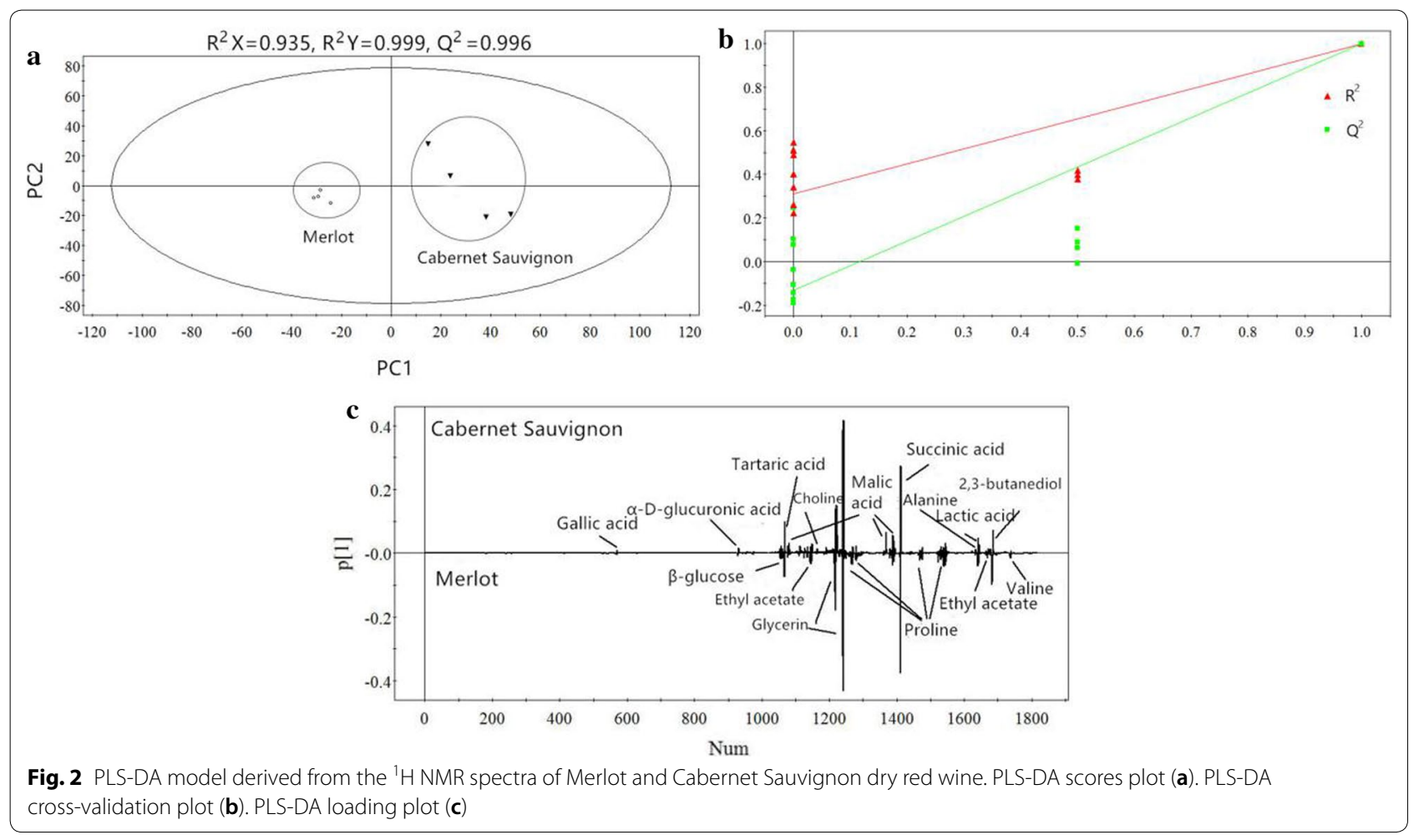


reliable. The permutation test was a widely used and effective method to verify the model quality. Results of cross-validation plot indicated that the values of $R^{2}$ and $\mathrm{Q}^{2}$ did not exceed the quality parameters of the actual model, once again demonstrating this PLS-DA model was of good reliability and predictability. Major metabolites that contributed to the discrimination of the two types of dry wine could be obtained from the PLS-DA loading plot. In the loading plot, the higher peak indicated higher content of the corresponding metabolites, while the lower one indicated lower content of the corresponding metabolites [13]. As seen from the loading plot, compared with Cabernet Sauvignon dry red wine, Merlot dry red wine had higher levels of proline, ethyl acetate, valine, 2,3-butanediol, succinic acid, glycerol, but lower levels of lactic acid, $\beta$-glucose, malic acid, tartaric acid, $\alpha-\mathrm{D}$-glucuronic acid, choline, alanine and gallic acid.

The PLS-DA scores plot, cross-validation plot and loading plot of Merlot and Cabernet Gernischt dry red wine was shown in Fig. 3. As seen from the scores plot, the two types of dry red wine were clearly distinguished on the $\mathrm{PC} 1$ axis, and the cumulative contribution rate $R^{2} X=0.943, R^{2} Y=0.998, Q^{2}=0.996$, indicating that this model was of good quality. As seen from the cross-validation plot of the permutation test in PLS-DA model, the values of $R^{2}$ and $Q^{2}$ did not exceed the quality parameters of the actual model, further demonstrated that the reliability and predictability of the model were better. It could be found from the loading plot that compared with Merlot dry red wine, Cabernet Gernischt dry red wine contained higher levels of choline, valine, malic acid, alanine, tartaric acid, ethyl acetate, lactic acid, proline, but lower levels of 2,3-butanediol, succinic acid, $\alpha$-DGlucuronic acid, glycerol and gallic acid.

The PLS-DA scores plot, cross-validation plot and loading plot of Cabernet Sauvignon and Cabernet Gernischt dry red wine was shown in Fig. 4. As seen from the scores plot, the two types of dry red wine were clearly distinguished on the $\mathrm{PC} 1$ axis, and the cumulative contribution rate $R^{2} X=0.965, R^{2} Y=0.999, Q^{2}=0.997$, indicating that the model was valid. As seen from the cross-validation plot of the permutation test in PLS-DA model, the values of $R^{2}$ and $Q^{2}$ did not exceed the quality parameters of the actual model, further illustrated that the reliability and predictability of the model were excellent. It could be found from the loading plot that compared with Cabernet Gernischt dry red wine, Cabernet Sauvignon dry red wine had higher levels of 2,3-butanediol, choline, tartaric acid, succinic acid, glycerol, gallic acid, $\alpha$-D-glucuronic acid, but lower levels of proline, ethyl acetate, lactic acid, valine, alanine and malic acid.

The main metabolites of these three wines were quantitatively analyzed and the results were shown in Table 2 . The content of these metabolites could be obtained by

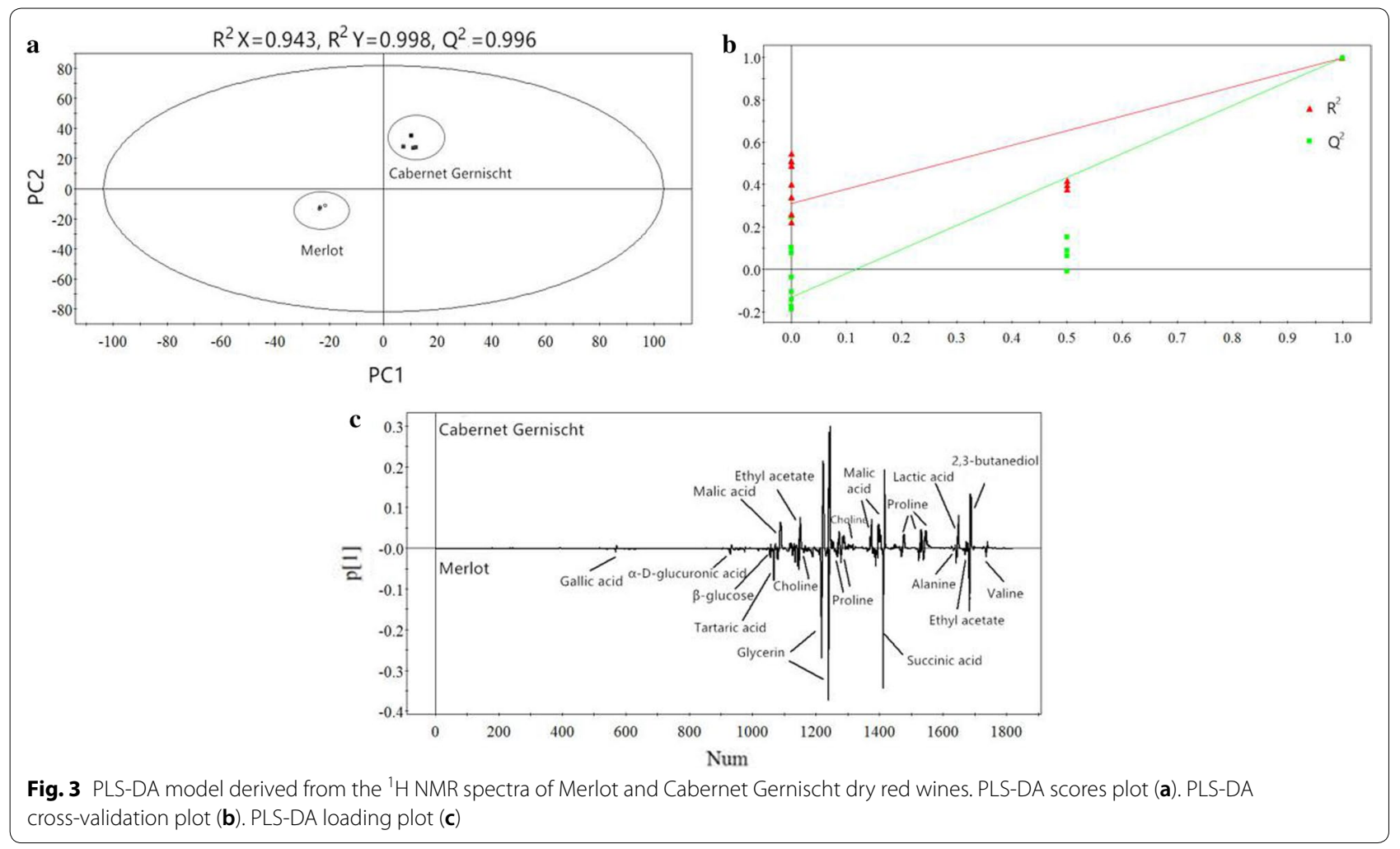



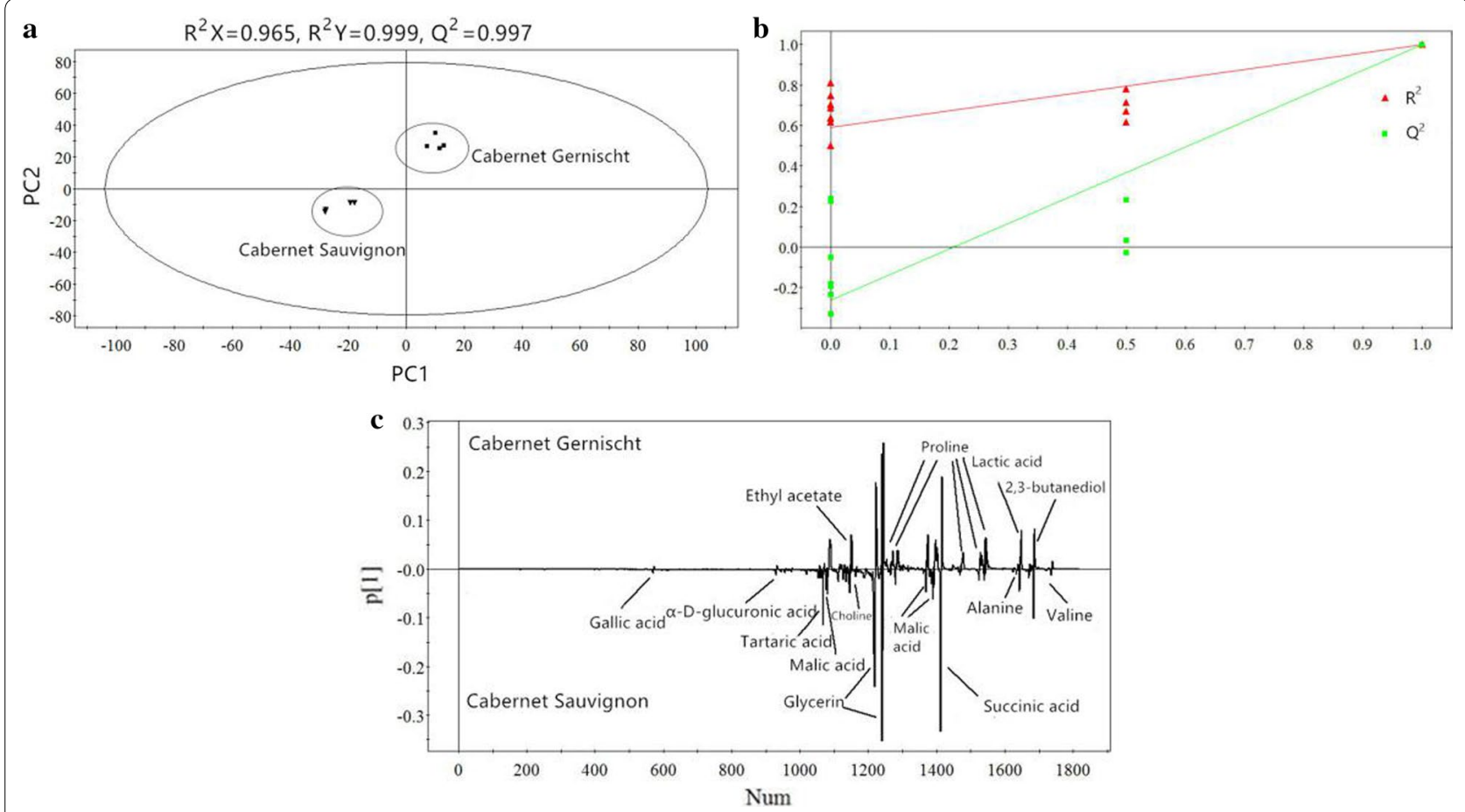

Fig. 4 PLS-DA model derived from the ${ }^{1}$ H NMR spectra of Cabernet Sauvignon and Cabernet Gernischt dry red wines. PLS-DA scores plot (a). PLS-DA cross-validation plot (b). PLS-DA loading plot (c)

Table 2 Content of the main metabolites in Merlot, Cabernet Sauvignon, and Cabernet Gernischt dry red wines (g/L)

\begin{tabular}{lccc}
\hline Metabolites & \multicolumn{2}{l}{ Contents $(\overline{\boldsymbol{x}} \pm \boldsymbol{S} \boldsymbol{N}, \boldsymbol{n = 4})$} \\
\cline { 2 - 4 } & Merlot & $\begin{array}{l}\text { Cabernet } \\
\text { Sauvignon }\end{array}$ & $\begin{array}{l}\text { Cabernet } \\
\text { Gernischt }\end{array}$ \\
\hline Ethyl acetate & $1.706 \pm 0.010^{\mathrm{a}}$ & $1.519 \pm 0.028^{\mathrm{b}}$ & $1.744 \pm 0.008^{\mathrm{a}}$ \\
Lactic acid & $0.459 \pm 0.015^{\mathrm{c}}$ & $0.508 \pm 0.029^{\mathrm{b}}$ & $0.622 \pm 0.030^{\mathrm{a}}$ \\
Alanine & $0.016 \pm 0.001^{\mathrm{c}}$ & $0.037 \pm 0.001^{\mathrm{b}}$ & $0.065 \pm 0.004^{\mathrm{a}}$ \\
Succinic acid & $1.440 \pm 0.027^{\mathrm{a}}$ & $1.312 \pm 0.016^{\mathrm{b}}$ & $1.122 \pm 0.054^{\mathrm{c}}$ \\
Proline & $3.891 \pm 0.157^{\mathrm{b}}$ & $3.226 \pm 0.044^{\mathrm{c}}$ & $4.311 \pm 0.081^{\mathrm{a}}$ \\
Malic acid & $4.076 \pm 0.240^{\mathrm{b}}$ & $4.954 \pm 0.166^{\mathrm{a}}$ & $5.022 \pm 0.0275^{\mathrm{a}}$ \\
Glycerol & $14.776 \pm 0.197^{\mathrm{a}}$ & $13.989 \pm 0.092^{\mathrm{b}}$ & $13.245 \pm 0.275^{\mathrm{c}}$ \\
Gallic acid & $0.113 \pm 0.002^{\mathrm{b}}$ & $0.135 \pm 0.003^{\mathrm{a}}$ & $0.102 \pm 0.004^{\mathrm{b}}$
\end{tabular}

All statistical analyses were performed using SPSS 17.0 software (SPSS Inc., Chicago, USA). Data are presented as the mean \pm SEM. The results were analysed by one-way ANOVA followed by Tukey's HSD post hoc test. A p-value $<0.05$ was considered statistically significant

calculating the ratio of the peak area generated by the proton on a specified group of the test substance to that of the added internal standard DSS in the one-dimensional ${ }^{1} \mathrm{H}$-NMR spectrum. As seen from the Table 2, the main metabolite content was significantly different, which was consistent with the PLS-DA model. The contents of succinic acid and glycerol in Merlot wine were the highest, gallic acid content in Cabernet Sauvignon wine was the highest, and the contents of lactic acid, alanine, proline and malic acid in Cabernet Gernischt wine were the highest. This finding could offer advice for the consumers to choose the suitable wine for their needs.

\section{Discussion}

Since the ${ }^{1} \mathrm{H}-\mathrm{NMR}$ measurement requires almost no sample pretreatment, the inherent properties of the sample are well retained. A large number of studies have demonstrated that metabolites detected by ${ }^{1} \mathrm{H}-\mathrm{NMR}$ can be used to non-destructive identification of wine varieties. Our results showed that there was little difference in the composition of metabolites in different varieties of dry red wines, while the content of these metabolites was quite different. The principal components responsible for the differences were ethyl acetate, lactic acid, alanine, succinic acid, proline, malic acid, glycerin, and gallic acid. These metabolites were significant, whose content were closely related to the flavor, taste and functional activity of wine.

Glycerol is the most abundant byproduct from yeasts alcoholic fermentation [20]. It has a slightly sweet taste and a viscous nature, which contributes to mouth-feel perception [21]. Several parameters, including $\mathrm{pH}$, temperature, sulfite concentration and yeast strain, have been shown to influence the final glycerol levels in wine 
$[14,22]$. In this experiment, the brewing process of different varieties of wine is the same, so the difference in glycerin content is mainly attributed to different wine varietals. The glycerol level of Merlot wine is higher than that of other wines, indicating that Merlot wine may has the richest mellow taste. Ethyl acetate accounts for more than $90 \%$ of the total acetate esters produced by yeast. Through the introduction of fruity and floral notes, it can make positive contributions to wine aroma [23]. This experiment showed that the ethyl acetate level in Merlot and Cabernet Gernischt wine are significantly higher than that in Cabernet Sauvignon wine. Therefore, flavor of Merlot and Cabernet Gernischt wine in this experiment may be more abundant than Cabernet Sauvignon wine. Succinic acid is quantitatively the second most important non-volatile byproduct of alcohol fermentation [23]. It has the strongest acidic reaction among the organic acids in the wine, which makes the wine rich in flavor and mellow in taste [24]. The content of succinic acid is affected by malic acid and amino acid concentrations and yeast species. Merlot wine has the highest succinic acid level in this experiment, and this result also supports the flavor and taste of Merlot is the most abundant. In addition, Adrian et al. [25] found that the concentration of glycerol and succinic acid have a strong correlation in a number of red wines, which was consistent with our results.

In terms of taste, the decrease in succinic acid gives wine a softer mouthfeel [26]. Cabernet Gernischt wine has the lowest succinic acid level in this experiment, indicating that Cabernet Gernischt wine may has the softest taste. Lactic acid is a gentle and soft acid, which mainly derived from malolactic fermentation process [27]. The content of lactic acid in Cabernet Gernischt wine is higher than that in other wines, which contributes to the gentle and soft taste of Cabernet Gernischt wine. In addition to giving the wine a flavour of acid, sweetness and bitterness, amino acids can also interact with ethanol, organic acids, sugars to form a unique mouthfeel, which will improve the nutritional value of the wine [26]. The proline content may be important for the wine's "mouth" or "body", because a salivary protein, which has a strong affinity for polyphenols, is rich in proline [14]. Chang et al. [28] found that the increase in the concentration of proline was beneficial to the balance of the wine. Lee et al. [12] found that the content of proline in wine depended on environmental factors and the variety of wine grape fruit. Alanine is an essential substance for the growth of yeast, and it exhibits a low content due to a large amount of consumption during the fermentation process. Herna'ndez-Orte et al. [29] found that addition of alanine and other three amino acids to grape juice of the Merlot variety can significantly decrease sulphured notes. Both of proline and alanine can soften the taste of the wine, and their contents in Cabernet Gernischt wine are the highest, further illustrated that Cabernet Gernischt wine has the sofetest taste.

Gallic acid, the most abundant phenolic compound in wine [30], is mainly derived from grape seeds and grape stems, as well as in contact with oak during fermentation process [14]. In this experiment, the gallic acid content of Cabernet Sauvignon wine is significantly higher than that of Merlot and Cabernet Gernischt wines. Researches have shown that gallic acid has anti-tumor effects and can resist a variety of carcinogenic substances [31]. Therefore, the bioactivity and health function of Cabernet Sauvignon wine in this experiment may be the highest among the three types of wines. Malic acid is an important organic acid in dry red wine, which are inherent in grape berry [32]. A large number of studies have shown that malic acid has many important biological activities, for example, it can effectively improve the body's exercise capacity, anti fatigue, accelerate the metabolism of carboxylate, protect the heart, and improve memory, etc. Malic acid content in Cabernet Sauvignon is higher, this result also supports that the bioactivity and health function of Cabernet Sauvignon wine in this experiment may be the highest among the three types of wines.

In this study, samples of Cabernet Sauvignon, Merlot and Cabernet Gernischt dry red wines vinified at Changli of Hebei province in 2017 were analyzed by ${ }^{1} \mathrm{H}$ NMR and multivariate statistical analysis methods. The main contributors to the notable differences among these three types of wines were identified. It provides a new technical solution for the adulteration identification of wine varieties, and also offers advice for the consumers to choose these three wines according to their personal preferences: they can choose Merlot wine in pursuit of the richer flavor and taste, Cabernet Gernischt wine in pursuit of the softer taste, and Cabernet Sauvignon wine in pursuit of the better healthy function.

\section{Abbreviations \\ ${ }^{1} \mathrm{H}$-NMR: Proton-nuclear magnetic resonance technique; PLS-DA: Partial least squares discrimination analysis; NMR: Nuclear magnetic resonance.}

\section{Authors' contributions \\ Formal analysis: ZX; Funding acquisition: FX; Methodology: ZJ; Validation: XS; Writing-original draft: GJ; Writing-review \& editing: HB. All authors read and approved the final manuscript.}

\section{Funding}

We acknowledge financial support by the National Natural Science Foundation of China. Project No. 31271857.

\section{Ethical approval}

This article does not contain any studies with human participants or animals performed by any of the authors. 


\section{Informed consent}

Not applicable.

\section{Competing interests}

The authors declare no Competing interests.

\section{Author details}

${ }^{1}$ The School of Tourism and Colinary Arts (The School of Food Science and Engineering), Yangzhou University, 196 Huayang West Road, Yangzhou 225127, Jiangsu, China. ${ }^{2}$ The Gradute School of East Asian Studies, Yamaguchi University, 1677-1 Yoshida, Yamaguchi-shi, Yamaguchi 753-8511, Japan. ${ }^{3}$ Kweichow Maotai Distillery (Group) Changli Winery Co., Ltd., 066600, East industrial Park, Changli, Qinhuangdao, Hebei, China.

Received: 17 January 2020 Accepted: 5 May 2020

Published online: 12 May 2020

\section{References}

1. Goldsmith P, Fenton H, Morris-Stiff G, Ahmad N, Fisher J, Prasad KR (2010) Metabonomics: a useful tool for the future surgeon. J Surg Res 160(1):122-132

2. Ogrinc N, Kosir IJ, Spangenberg JE, Kidrič J (2003) The application of NMR and MS methods for detection of adulteration of wine, fruit juices and olive oil. A review. Anal Bioanal Chem 376(4):424-430

3. Lopez-Rituerto E, Avenoza A, Busto JH, Peregrina JM (2010) Evidence of metabolic transformations of amino acids into higher alcohols through 13C NMR studies of wine alcoholic fermentation. J Agric Food Chem 58(8):4923-4927

4. Duarte I, Barros A, Belton PS, Righelato R, Spraul M, Humpfer E, Gil AM (2002) High-resolution nuclear magnetic resonance spectroscopy and multivariate analysis for the characterization of beer. J Agric Food Chem 50(9):2475-2481

5. Lee JE, Lee BJ, Chung JO, Shin HJ, Lee SJ, Lee CH, Hong YS (2011) NMRbased metabolomic characterization during green tea (Camellia sinensis) fermentation. Food Res Int 44(2):597-604

6. Ali K, lqbal M, Fortes AM, Pais Maria Salomé, Korthout HAAJ, Verpoorte R, Choi YH (2013) Red wines attenuate TNF production in human histiocytic lymphoma cell line: an NMR spectroscopy and chemometrics based study. Food Chem 141(3):3124-3130

7. Mazzei P, Francesca N, Moschetti G, Piccolo A (2010) NMR spectroscopy evaluation of direct relationship between soils and molecular composition of red wines from Aglianico grapes. Anal Chim Acta 673(2):167-172

8. Belton PS, Colquhoun IJ, Kemsley EK, Delgadillo I, Roma P, Dennis MJ, Sharman M, Holmes E, Nicholson JK, Spraul M (1998) Application of chemometrics to the ${ }^{1} \mathrm{H}$ NMR spectra of apple juices: discrimination between apple varieties. Food Chem 61(1-2):207-213

9. Pereira GE, Gaudillere JP, Leeuwen CV, Hilbert G, Maucourt M, Deborde C, Moing A, Rolin D (2006) ${ }^{1} \mathrm{H}$ NMR metabolite fingerprints of grape berry: comparison of vintage and soil effects in Bordeaux grapevine growing areas. Anal Chim Acta 563(1-2):346-352

10. Viggiani L, Morelli MAC (2008) Characterization of wines by nuclear magnetic resonance: a work study on wines from the Basilicata Region in Italy. J Agric Food Chem 56(18):8273-8279

11. Héberger K, Csomós E, Simon-Sarkadi L (2003) Principal component and linear discriminant analyses of free amino acids and Biogenic Amines in Hungarian Wines. J Agric Food Chem 51 (27):8055-8060

12. Lee JE, Hwang GS, Van Den Berg F, Lee CH, Hong YS (2009) Evidence of vintage effects on grape wines using ${ }^{1} \mathrm{H}$ NMR-based metabolomic study. Anal Chim Acta 648(1):71-76

13. Zhu J, Hu B, Lu J, Xu S (2018) Analysis of metabolites in Cabernet Sauvignon and Shiraz Dry Red Wines from Shanxi by ${ }^{1} \mathrm{H}$ NMR spectroscopy combined with pattern recognition analysis. Open Chem 16(1):446-452

14. Son HS, Kim KM, Van Den Berg F, Hwang GS, Park WM, Lee CH, Hong YS (2008) ${ }^{1} \mathrm{H}$ Nuclear magnetic resonance-based metabolomic characterization of wines by grape varieties and production areas. J Agric Food Chem 56(17):8007-8016

15. Anastasiadi M, Zira A, Magiatis P, Haroutounian SA, Mikros E (2009) ${ }^{1} \mathrm{H}$ NMR-Based metabonomics for the classification of Greek wines according to variety, region, and vintage. Comparison with HPLC Data. J Agric Food Chem 57(23):11067-11074

16. Son HS, Hwang GS, Kim KM, Ahn HJ, Park WM, Van Den Berg F, Hong YS, Lee $\mathrm{CH}$ (2009) Metabolomic studies on geographical grapes and their wines using ${ }^{1} \mathrm{H}$ NMR analysis coupled with multivariate statistics. J Agric Food Chem 57(4):1481-1490

17. Fotakis C, Kokkotou K, Zoumpoulakis P, Zervou M (2013) NMR metabolite fingerprinting in grape derived products: an overview. Food Res Int 54(1):1184-1194

18. Hu B, Cao Y, Zhu J, Xu W, Wu W (2019) Analysis of metabolites in chardonnay dry white wine with various inactive yeasts by ${ }^{1} \mathrm{H}$ NNR spectroscopy combined with pattern recognition analysis. AMB Express 9(1):140

19. Hu B, Yue Y, Zhu Y, Wen W, Zhang F, Hardie JW (2015) Proton nuclear magnetic resonance-spectroscopic discrimination of wines reflects genetic homology of several different grape (V. vinifera L.) cultivars. PLOS ONE 10(12):e0142840

20. Liu S, Laaksonen O, Kortesniemi M, Kalpio M, Yang B (2018) Chemical composition of bilberry wine fermented with non-saccharomyces yeasts (Torulaspora delbrueckii and Schizosaccharomyces pombe) and Saccharomyces cerevisiae in pure, sequential and mixed fermentations. Food Chem 266:262-274

21. Nieuwoudt HH, Prior BA, Pretorius IS, Bauer FF (2002) Glycerol in South African Table Wines: an assessment of its relationship to wine quality. $S$ Afr J Enol Vitic 23(1):22-30

22. Yalcin SK, Ozbas ZY (2005) Determination of growth and glycerol production kinetics of a wine yeast strain Saccharomyces cerevisiae Kalecik 1 in different substrate media. World J Microbiol Biotechnol 21:1303-1310

23. Yan G, Zhang B, Joseph L, Waterhouse AL (2020) Effects of initial oxygenation on chemical and aromatic composition of wine in mixed starters of Hanseniaspora vineae and Saccharomyces cerevisiae. Food Microbiol 90:103460

24. Lamikanra Olusola (1997) Changes in organic acid composition during fermentation and aging of noble muscadine. Wine J Agric Food Chem 45(3):935-937

25. Coulter AD (2004) Succinic acid-how it is formed, what is its effect on titratable acidity, and what factors influence its concentration in wine? Wine Ind J 19:16-25

26. Zhang XK, Lan YB, Zhu BQ, Xiang XF, Duan CQ, Shi Y (2018) Changes in monosaccharides, organic acids and amino acids during Cabernet Sauvignon wine aging based on a simultaneous analysis using gas chromatography-mass spectrometry. J Sci Food Agric 98(1):104-112

27. Zheng YJ, Duan YT, Zhang YF, Pan OH, Li JM, Huang WD (2009) Determination of organic acids in red wine and must on only one RP-LCColumn directly after sample dilution and filtration. Chromatographia 69(11-12):1391-1395

28. Chang EH, Jung SM, Park SJ, Noh JH, Hur YY, Nam JC, Park KS (2014) Wine quality of grapevine 'cheongsoo' and the related metabolites on proton nuclear magnetic resonance $(\mathrm{nmr})$ spectroscopy at the different harvest times. Plant Omics 7(2):80-86

29. Hernández-Orte P, Ibarz MJ, Cacho J, Ferreira V (2006) Addition of amino acids to grape juice of the merlot variety: effect on amino acid uptake and aroma generation during alcoholic fermentation. Food Chem 98(2):300-310

30. Issa-Issa H, Guclu G, Noguera-Artiaga L, Lopez-Lluch D, Poveda R, Kelebek H, Selli S, Carbonell-Barrachina ÁA (2020) Aroma-active compounds, sensory profile, and phenolic composition of Fondillón. Food Chem 316:126353

31. Silva I, Campos FM, Hogg T, Couto JA (2011) Wine phenolic compounds influence the production of volatile phenols by wine-related lactic acid bacteria. J Appl Microbiol 111(2):360-370

32. Liu HF, Wu BH, Fan PG, Li SH, Li LS (2006) Sugar and acid concentrations in 98 grape cultivars analyzed by principal component analysis. J Sci Food Agric 86:1526-1536

\section{Publisher's Note}

Springer Nature remains neutral with regard to jurisdictional claims in published maps and institutional affiliations. 